\title{
Traducción y validación de la versión española de la Échelle des Perceptions du Soutien à l'Autonomie en Sport*
}

Translation and validation of the Spanish versión of the Échelle des Perceptions du Soutien à l'Autonomie en Sport

Recibido: enero 20 de 2010 | Revisado: mayo 20 de 2010 | Aceptado: septiembre 26 de 2010

\author{
JAIME LEÓN ** \\ JUAN L. NÚÑEZ *** \\ Evelia DOMÍNGUEZ \\ PATRICIA MarTín \\ Universidad de las Palmas de Gran Canaria, España \\ JosÉ MARTÍN-ALBO \\ Universidad de Zaragoza, España
}

RES U MEN

El objetivo de este estudio fue traducir y analizar las propiedades psicométricas de la versión española de la Échelle des Perceptions du Soutien à l'Autonomie en Sport, en un contexto deportivo. Los resultados apoyaron la estructura unifactorial de la escala. Asimismo, el modelo de ecuaciones estructurales mostró evidencias de validez concurrente, en el que la percepción del apoyo a la autonomía predice de manera positiva la percepción de autonomía y la percepción de competencia y estas, a su vez, predicen la motivación intrínseca, que muestra un efecto positivo sobre las emociones positivas durante la práctica deportiva. Finalmente, se muestran evidencias de fiabilidad. Los resultados apoyan preliminarmente el uso de la versión española en el contexto deportivo.

Palabras clave autores:

Autodeterminación, deporte, percepción de apoyo a la autonomía, validación. Palabras clave descriptores:

Pruebas psicológicas, psicología del deporte, investigación cuantitativa, ecuaciones estructurales.

\section{A B S T R A C T}

Domínguez, E., Martín, P. \& Martín-Albo, J. (2012). Traducción y validación de la versión española de la Échelle des Perceptions du Soutien à l'Autonomie en Sport. Universitas Psychologica, 11(1), 165-175.

* Artículo de investigación en psicometría

** Departamento de Educación. C/ Santa Juana de Arco, 1. 35004. Las Palmas. E-mail: jleon@dedu. ulpgc.es

***: Departamento de Psicología y Sociología. C/ Santa Juana de Arco, 1. 35004. Las Palmas. E-mail: jnunez@dps.ulpgc.es. Researcher-ID: Núñez, J. L., B-4513-2010
The aim of the present research was to translate and to analyze the psychometric properties of the Spanish version of the Échelle des Perceptions du Soutien à l'Autonomie en Sport in sport context. Results supported the unidimentional structure of the scale. The predictive validity was tested using a structural equation model which perceived autonomy support predicted the perceived autonomy and perceived competence and these two the intrinsic motivation. Finally, intrinsic motivation predicted positive emotions. Likewise, results showed evidences of reliability. Results support preliminary the use of the Spanish version of the scale in sport context. Key words authors:

Self-determination, sport, perceived autonomy support, validation.

Key words plus:

Psychological test, sport psychology, quantitative research, structural equations. 
En los últimos 25 años se ha producido un interés creciente de la comunidad científica por el estudio del apoyo a la autonomía, debido a su relación con variables como satisfacción con la vida y autoestima (Philippe \& Vallerand, 2008), persistencia en la práctica deportiva (Pelletier, Fortier, Vallerand \& Brière, 2001), concentración (Ntoumanis, 2005) y depresión (Wong \& Wiest, 1999). El apoyo a la autonomía también ha demostrado su importancia en varios contextos, como en el campo de la salud (Williams, Freedman \& Deci, 1998), el laboral (Deci et al., 2001), la educación física (Moreno, Parra \& González-Cutre, 2008) y el deporte (Álvarez, Balaguer, Castillo \& Duda, 2009).

Deci y Ryan $(1985,1991)$ describen el apoyo a la autonomía como un contexto en el que se promueve la elección, se minimiza la presión para ejecutar las tareas de una manera determinada y se fomenta la iniciativa. Para Hackman y Oldham (1975) el apoyo a la autonomía es una dimensión importante que explica como influyen las características del trabajo en la motivación del trabajador y lo definen como la oportunidad para la libertad, la independencia y la discreción.

Algunas de las características que definen el concepto de apoyo a la autonomía son los siguientes: que el superior (profesor, entrenador, etc.) entienda y acepte las decisiones de sus inferiores (alumno, deportista, etc.), aporte información con sentido, sugiera alternativas (Deci et al., 2001) y que proporcione una retroalimentación no controladora (Deci, Connell \& Ryan, 1989). Tanto Reeve (2002) como Hagger et al. (2007) indican que el efecto determinante del apoyo a la autonomía es la interpretación que el inferior realiza sobre como su superior apoya la autonomía. Para entender mejor este hecho, habría que tener en cuenta la influencia que tiene lo que se percibe en términos cognitivos y conductuales, independientemente de que estas percepciones sean acertadas o no (Bandura, 1997; Petrides \& Furham, 2003). Es por esto que se debería evaluar el apoyo a la autonomía percibido por el subordinado.

En el contexto deportivo, la percepción de apoyo a la autonomía se define como las creencias de los deportistas de que su entrenador apoya las iniciativas propias, permite diferentes posibilidades a la hora de elegir una tarea, fomenta la resolución de problemas de una manera individual y la participación en la toma de decisiones, además de reconocer los sentimientos y evitar las presiones (Hagger et al., 2007; Mageau \& Vallerand, 2003).

La percepción de apoyo a la autonomía ha sido evaluada con múltiples instrumentos. En el campo de la salud, se ha medido con el HealthCare Climate Questionnaire (Williams, Grow, Freedman, Ryan \& Deci, 1996), en la educación con el Learning Climate Questionnaire ([LCQ], Williams \& Deci, 1996), en el trabajo con el Work Climate Questionnaire ([WCQ], Baard, Deci \& Ryan, 2004) y en el deporte de equipo con el Sport Climate Questionnaire ([SCQ], SDT página web http://www.psych.rochester.edu/SDT/), recientemente traducido y validado al español por Balaguer, Castillo, Duda y Tomás (2009).

Hagger et al. (2007) desarrollaron otro instrumento denominado Perceived Autonomy Support Scale for Exercise Settings (PASSES). Esta escala fue traducida al español y validada en el contexto de la educación física por Moreno et al. (2008). También fue traducida al francés y adaptada al contexto deportivo por Gillet, Vallerand, Paty, Gobancé y Berjot (en prensa), con el nombre Échelle des Perceptions du Soutien à l'Autonomie en Sport (EPSAS). La escala consta de 12 ítems agrupados en un factor y se puntúan mediante una escala tipo Likert desde 1 (totalmente en desacuerdo) a 7 (totalmente de acuerdo). La consistencia interna medida con el alfa de Cronbach fue de 0.91 y la estabilidad temporal después de 2 meses medida con la correlación test-retest fue de 0.71 . Todos los ítems tuvieron un peso factorial por encima de 0.64 y los índices de ajuste también fueron adecua$\operatorname{dos}\left(\chi^{2}=119.94, g l=54, p<0.01 ;\right.$ CFI $=0.97$; $\mathrm{NNFI}=0.95 ;$ RMSEA $=0.05 ; \quad(\mathrm{LO} 90=0.04$; HI $90=0.07$ ); SRMR $=0.04$ ).

Según el modelo jerárquico de motivación intrínseca y extrínseca (Vallerand, 1997), el apoyo a la autonomía se considera un factor social que influye sobre la motivación, a través de la mediación de las necesidades psicológicas básicas (percepción de autonomía, percepción de competencia y percepción de relaciones sociales). En este sentido, la percepción 
de apoyo a la autonomía se ha considerado como un determinante de la percepción de autonomía (necesidad de sentirse el origen de las acciones) y la percepción de competencia (necesidad de sentirse eficaz y competente a la hora de ejecutar tareas), ya que, minimizando el control se fomenta la autonomía y facilitando aspectos informativos de las tareas, se aumenta el sentimiento de competencia (Reeve \& Deci, 1996). Tal y como indica la teoría de la evaluación cognitiva propuesta por Deci y Ryan (1985), los factores sociocontextuales que promueven sentimientos de autonomía y competencia facilitan la motivación intrínseca (Gagné \& Deci, 2005). La motivación intrínseca se refiere al compromiso en una actividad por el placer y satisfacción que se derivan de su práctica (Deci \& Ryan, 1985; Ryan $\&$ Deci, 2000). Un deportista está intrínsecamente motivado cuando practica deporte únicamente por el placer que siente, por la satisfacción de aprender y de superarse constantemente en su deporte.

Vallerand, Blais, Brière y Pelletier (1989) consideran la motivación intrínseca como un constructo multidimensional en el que se distinguen tres tipos: motivación intrínseca hacia el conocimiento, que implica llevar a cabo una conducta por el placer y satisfacción que se experimentan al aprender cosas nuevas, explorar o intentar comprender, por ejemplo, nuevas técnicas de entrenamiento; motivación intrínseca hacia el logro, que ha sido denominada también motivación orientada a la maestría y se refiere a ejecutar una actividad por la satisfacción de intentar conseguir o dominar, por ejemplo, nuevos elementos técnicos o tácticos; y motivación intrínseca hacia las experiencias estimulantes, que ocurre cuando se realiza una tarea para experimentar las sensaciones estimulantes asociadas a ella, por placer o para vivir experiencias excitantes. La motivación intrínseca ha sido un elemento importante para la explicación del bienestar (Vallerand \& Losier, 1999), tanto el bienestar hedónico, afectos positivos que se derivan de poder hacer o tener lo que uno quiere, como el bienestar eudaimónico, sentimientos de bienestar derivados de la autorrealización (Waterman, Schwartz \& Conti, 2008). En este estudio, se ha considerado estudiar el bienestar hedónico a través de las emociones positivas, ya que en el contexto deportivo el bienestar de los deportistas puede ser considerado una consecuencia de la percepción de autonomía (Blanchard, Amiot, Perreault, Vallerand \& Provencher, 2009).

Puesto que la EPSAS se ha mostrado como un instrumento adecuado para evaluar la percepción del apoyo a la autonomía en el contexto deportivo, y teniendo en cuenta que no existe disponible una versión traducida al español y validada en dicho contexto, los objetivos de este estudio son: a) traducir al español la EPSAS; b) analizar la evidencia de validez de constructo a través de un análisis factorial confirmatorio (AFC); c) analizar la evidencia de fiabilidad a través de la consistencia interna y la estabilidad temporal; d) analizar la evidencia de validez concurrente probando un modelo de ecuaciones estructurales donde analizaremos la relación entre el apoyo a la autonomía, la percepción de competencia y la percepción de autonomía, la motivación intrínseca y las emociones positivas.

\section{Método}

\section{Participantes}

Formaron parte de este estudio un total 326 deportistas, 248 hombres y 78 mujeres, que practicaban diferentes deportes (fútbol, baloncesto, hockey, kárate, waterpolo, balonmano, voleibol, natación y tenis). La media de edad de los participantes fue de 20.25 años $(D E=4.75)$ y el rango de edad se situó entre 14 y 35 años.

Para el análisis de la estabilidad temporal de la escala, se utilizó una segunda muestra de 54 deportistas (practicantes de futbol, baloncesto y waterpolo), 34 hombres y 20 mujeres, con una media de edad de 21.11 años $(D E=4.83)$ que cumplimentaron la escala en dos ocasiones, tras un intervalo de ocho semanas.

\section{Instrumentos}

Para medir la percepción de apoyo a la autonomía se utilizó la EPSAS (Gillet et al., en prensa). Para traducir esta escala al español se adoptó la estrategia de traducción inversa (Hambleton, 1996). Se tradujeron 
los ítems al español y posteriormente otro grupo de traductores volvió a traducirlo al francés, comparándolos con los originales. Posteriormente, se sometieron los ítems a una evaluación por parte de tres expertos en motivación deportiva, de acuerdo con Lynn (1986), que estimaron la adecuación de los ítems al constructo al que se referían. La escala traducida ha pasado a denominarse Escala de Percepción de Apoyo a la Autonomía en Contextos Deportivos (EPAAD). Este instrumento consta de 12 ítems agrupados en un único factor que se puntúan de acuerdo a una escala tipo Likert de 7 puntos desde 1 (nada de acuerdo) a 7 (totalmente de acuerdo), con una puntuación intermedia de 4 (medianamente de acuerdo).

La percepción de autonomía y la percepción de competencia se midieron utilizando dos subescalas de la Basic Need Satisfaction in Sport Scale (Gillet, Rosnet \& Vallerand, 2008) de cinco ítems cada una. Se puntúan de acuerdo a una escala tipo Likert, desde 1 (nada cierto) a 7 (muy cierto). La consistencia interna y los índices de ajuste para la subescala percepción de autonomía fueron: $\alpha=0.76, \chi^{2} /$ $g l=1.32 \mathrm{IFI}=0.99, \mathrm{CFI}=0.99, \mathrm{RMSEA}=0.03$ $(\mathrm{LO} 90=0.00 ; \mathrm{HI} 90=0.09)$ y $\mathrm{SRMR}=0.01$ y para la subescala percepción de competencia: $\alpha=0.77, \chi^{2} / g l=0.16$, IFI $=0.99$, CFI $=0.99$, RMSEA $=0.01($ LO $90=0.00 ; \mathrm{HI} 90=0.06) \mathrm{y}$ $\operatorname{SRMR}=0.01$

La motivación intrínseca se evaluó empleando la versión traducida al español por Núñez, MartínAlbo, Navarro y González (2006) de la Sport Motivation Scale ([SMS], Pelletier et al., 1995). Se utilizaron los 12 ítems que miden la motivación intrínseca, encabezados por el enunciado "iPor qué practicas tu deporte?", que se responden de acuerdo a una escala tipo Likert de 1 (totalmente en desacuerdo) a 7 (totalmente de acuerdo). La consistencia interna y los índices de ajuste para la subescala de motivación intrínseca fueron: $\alpha=0.93, \chi^{2} / g l=5.52$, IFI $=0.90$, CFI $=0.90$, RMSEA $=0.11($ LO $90=0.10 ; \mathrm{HI} 90=0.13) \mathrm{y}$ $\mathrm{SRMR}=0.06$. Además, los valores alfa para cada una de las subescalas que componen la motivación intrínseca fueron de 0.88 para la motivación intrínseca al logro, 0.83 para la motivación intrínseca al conocimiento y 0.79 para la motivación intrínseca a las experiencias estimulantes.

Para medir el bienestar subjetivo se utilizó la subescala de emociones positivas del cuestionario Autonomy Perception in Life Context Scales (Blais \& Vallerand, 1991). Esta escala consta de cuatro ítems que contestan al enunciado: "Mientras practico mi deporte..." y se responden en una escala tipo Likert con puntuaciones desde 1 (nada de acuerdo) a 7 (completamente de acuerdo). La consistencia interna y los índices de ajuste para la subescala de emociones positivas fueron: $\alpha=0.89, \chi^{2} / g l=2.22, \mathrm{IFI}=0.99, \mathrm{CFI}=0.99$, RMSEA $=0.06($ LO $90=0.01 ;$ HI $90=0.13) y$ $\mathrm{SRMR}=0.01$.

\section{Procedimiento}

Se hizo contacto con los entrenadores de los diversos clubes deportivos para solicitar su autorización y explicarles los objetivos de la investigación. A aquellos deportistas menores de edad se les entregó una carta de información a los padres del motivo del estudio, solicitándoles su autorización. Los instrumentos se administraron en las instalaciones de cada club, antes del entrenamiento. Se comunicó a los deportistas que la participación era voluntaria y confidencial, enfatizando que no había respuestas correctas o incorrectas e instándoles a que la contestaran con honestidad. El tiempo empleado por los deportistas en responder a los cuestionarios, fue aproximadamente de 15 minutos.

\section{Análisis de datos}

En primer lugar, se llevó a cabo un análisis descriptivo de los ítems de la EPAAD. En segundo lugar, se realizó un análisis de correlaciones con las variables percepción de apoyo a la autonomía, necesidades psicológicas básicas (percepción de autonomía y percepción de competencia), los tres tipos de motivación intrínseca (hacia la ejecución, hacia la estimulación y hacia el conocimiento) y emociones positivas. En tercer lugar, para determinar la validez de constructo de la EPAAD, se llevó a cabo un AFC. En cuarto lugar, se realizó un análisis 
de fiabilidad a través de la consistencia interna y la estabilidad temporal. Por último, se analizó la evidencia de validez concurrente de la escala a partir de un modelo de ecuaciones estructurales. Para realizar estos análisis, se utilizaron los programas estadísticos AMOS 18 y SPSS 18.

\section{Resultados}

\section{Análisis descriptivos}

La Tabla 1 presenta los estadísticos descriptivos (media, desviación típica, asimetría y curtosis) de los ítems que han sido utilizados en este estudio. Los valores de asimetría y curtosis se situaron por debajo del valor absoluto 2 , lo que permite utilizar procedimientos de máxima verosimilitud, tal y como sugieren Curran, West y Finch (1996). Asimismo, el coeficiente de Mardia fue de 62.34, lo que supone un incumplimiento del supuesto de normalidad multivariada.

\section{Análisis de correlaciones entre la percepción de apoyo a la autonomía y otras variables}

Se analizó la relación entre la percepción de apoyo a la autonomía, la percepción de autonomía, la percepción de competencia, los tres tipos de motivación intrínseca (hacia el logro, hacia la estimulación y hacia el conocimiento) y las emociones positivas, con el coeficiente de correlación de Pearson. Los resultados mostraron que las correlaciones entre todas las variables fueron positivas y significativas $(p<0.01)$. Tal y como se observa en la Tabla 2 , el factor percepción de apoyo a la autonomía obtuvo la correlación más alta con el factor percepción de competencia $(r=0.49)$ y la más baja, con el factor emociones positivas $(r=0.26)$.

TABLA 1

Estadísticos descriptivos de los ítems de la EPAAD

\begin{tabular}{|c|c|c|c|c|c|}
\hline Ítems & Media & $\mathrm{DE}$ & Asimetría & Curtosis & $\begin{array}{l}\text { alfa sin el } \\
\text { ítem }\end{array}$ \\
\hline $\begin{array}{l}\text { 1. Creo que mi entrenador me deja tomar decisiones en } \\
\text { este deporte. }\end{array}$ & 4.35 & 1.76 & -0.18 & -0.79 & 0.92 \\
\hline $\begin{array}{l}\text { 2. Creo que mi entrenador entiende por qué he elegido } \\
\text { este deporte. }\end{array}$ & 4.99 & 1.58 & -0.60 & -0.19 & 0.92 \\
\hline $\begin{array}{l}\text { 3. Mi entrenador confía en mi capacidad para hacer este } \\
\text { deporte. }\end{array}$ & 5.42 & 1.44 & -0.81 & 0.18 & 0.92 \\
\hline $\begin{array}{l}\text { 4. Mi entrenador me anima a entregarme por completo } \\
\text { en este deporte. }\end{array}$ & 5.59 & 1.57 & -1.07 & 0.36 & 0.92 \\
\hline $\begin{array}{l}\text { 5. Mi entrenador me presta atención durante la práctica } \\
\text { deportiva. }\end{array}$ & 5.25 & 1.47 & -0.82 & 0.51 & 0.92 \\
\hline 6. Mi entrenador me apoya en este deporte. & 5.45 & 1.45 & -0.82 & 0.10 & 0.91 \\
\hline $\begin{array}{l}\text { 7. Puedo hablar sin problema de este deporte con mi } \\
\text { entrenador. }\end{array}$ & 5.85 & 1.45 & -1.31 & 10.08 & 0.92 \\
\hline $\begin{array}{l}\text { 8. Mi entrenador se preocupa de que yo entienda por qué } \\
\text { necesito practicar este deporte. }\end{array}$ & 4.83 & 1.58 & -0.53 & -0.28 & 0.92 \\
\hline $\begin{array}{l}\text { 9. Mi entrenador responde a mis dudas sobre este } \\
\text { deporte. }\end{array}$ & 5.64 & 1.50 & -1.09 & 0.56 & 0.92 \\
\hline $\begin{array}{l}\text { 10. Mi entrenador se interesa por mí durante la práctica } \\
\text { deportiva. }\end{array}$ & 5.34 & 1.39 & -0.65 & -0.10 & 0.91 \\
\hline $\begin{array}{l}\text { 11. Siento que puedo compartir mis experiencias } \\
\text { deportivas con mi entrenador. }\end{array}$ & 5.17 & 1.69 & -0.66 & -0.49 & 0.92 \\
\hline $\begin{array}{l}\text { 12. Confío en los consejos que me da mi entrenador sobre } \\
\text { este deporte. }\end{array}$ & 5.94 & 1.35 & -1.43 & 10.74 & 0.92 \\
\hline
\end{tabular}

Fuente: elaboración propia. 
TABLA 2

Estadísticos descriptivos, correlaciones entre las variables y alfa de Cronbach (en la diagonal)

\begin{tabular}{lccccccccccc}
\hline & Media & $D E$ & Asimetría & Curtosis & 1 & 2 & 3 & 4 & 5 & 6 & 7 \\
\hline Apoyo autonomía & 5.32 & 1.12 & -0.81 & 0.29 & 0.92 & 0.48 & 0.49 & 0.32 & 0.33 & 0.35 & 0.26 \\
Autonomía & 3.94 & 1.31 & 0.35 & -0.42 & & 0.76 & 0.46 & 0.34 & 0.36 & 0.31 & 0.25 \\
Competencia & 5.30 & 1.02 & -0.45 & -0.16 & & & 0.77 & 0.37 & 0.34 & 0.44 & 0.41 \\
MI logro & 5.22 & 1.39 & -0.75 & 0.13 & & & & 0.88 & 0.77 & 0.75 & 0.32 \\
MI conocimiento & 5.01 & 1.41 & -0.51 & -0.31 & & & & 0.83 & 0.69 & 0.41 \\
MI estimulantes & 5.56 & 1.12 & -0.82 & 0.64 & & & & & 0.79 & 0.4 \\
Emociones positivas & 6.21 & 1.00 & -1.43 & 1.90 & & & & & & & 0.89 \\
\hline
\end{tabular}

Fuente: elaboración propia.

\section{Análisis Factorial Confirmatorio}

La estructura factorial de la EPAAD fue evaluada con un AFC, utilizando la estimación de máxima verosimilitud y la matriz de covarianza entre los ítems como entrada para el análisis de datos. Puesto que el coeficiente de Mardia fue de 62.34, lo que indica una posible desviación del supuesto de normalidad, se procedió a realizar un bootstrapping standard con 500 remuestreos. Este procedimiento permite comparar los valores estimados sin bootstrapping con el conjunto de valores estimados en el mismo, indicando el nivel de sesgo. Los intervalos de confianza no deben incluir el valor cero, para rechazar la hipótesis nula que considera que los pesos factoriales son iguales a cero en la población (Byrne, 2001).

Como se observa en la Tabla 3, los resultados del bootstrapping mostraron que los errores típicos de los pesos factoriales oscilaron entre 0.04 y 0.08 y los niveles de sesgo fueron bajos. Además, los intervalos de confianza ajustados no incluyeron el valor cero, por lo que se puede afirmar que los parámetros estimados son distintos de cero en la población.

Todos los pesos de regresión estandarizados fueron significativos $(p<0.01)$ y oscilaron entre 0.54 del ítem 2 y 0.87 del ítem 6 . Los resultados obtenidos a través del AFC fueron: $\chi^{2} / g l=3.82$, IFI $=0.93$,

Tabla 3

Errores estandarizados de los factores en el bootstrapping e intervalos de confianza ajustados de los parámetros

\begin{tabular}{|c|c|c|c|c|c|c|c|c|c|}
\hline & Parámetros & SE & SE-SE & Media & Sesgo & SE-Sesgo & ICI & ICS & $p$ \\
\hline ítem 1 & $<$ <.- Apoyo Autonomía & 0.07 & 0.01 & 0.83 & 0.01 & 0.01 & 0.73 & 0.94 & 0.004 \\
\hline ítem 2 & <..- Apoyo Autonomía & 0.08 & 0.01 & 0.68 & 0.02 & 0.02 & 0.56 & 0.81 & 0.005 \\
\hline ítem 3 & <..- Apoyo Autonomía & 0.07 & 0.01 & 0.75 & 0.01 & -0.01 & 0.65 & 0.86 & 0.003 \\
\hline ítem 4 & <... Apoyo Autonomía & 0.05 & 0.01 & 1.01 & 0.01 & -0.01 & 0.91 & 1.08 & 0.004 \\
\hline ítem 5 & <..- Apoyo Autonomía & 0.06 & 0.01 & 0.87 & 0.01 & -0.01 & 0.77 & 0.97 & 0.004 \\
\hline ítem 6 & <..- Apoyo Autonomía & 0.01 & 0.01 & 1 & $\ldots$ & 0 & 1 & 1 & $\ldots$ \\
\hline ítem 7 & <..- Apoyo Autonomía & 0.06 & 0.01 & 0.84 & 0.02 & 0.01 & 0.73 & 0.94 & 0.005 \\
\hline ítem 8 & <..- Apoyo Autonomía & 0.06 & 0.01 & 0.87 & 0.02 & 0.01 & 0.77 & 0.96 & 0.006 \\
\hline ítem 9 & <..- Apoyo Autonomía & 0.06 & 0.01 & 0.79 & 0.02 & 0.01 & 0.7 & 0.9 & 0.005 \\
\hline ítem 10 & <..- Apoyo Autonomía & 0.04 & 0.01 & 0.92 & 0.01 & -0.01 & 0.85 & 0.99 & 0.004 \\
\hline ítem 11 & <... Apoyo Autonomía & 0.06 & 0.01 & 0.98 & 0.02 & 0.01 & 0.9 & 1.07 & 0.005 \\
\hline ítem 12 & <..- Apoyo Autonomía & 0.06 & 0.01 & 0.73 & 0.01 & 0.01 & 0.64 & 0.85 & 0.003 \\
\hline
\end{tabular}

Nota. SE = Error estandarizado; ICI = intervalo de confianza inferior; ICS = Intervalo de confianza superior Fuente: elaboración propia. 
$\mathrm{CFI}=0.93, \mathrm{RMSEA}=0.09(\mathrm{LO} 90=0.08 ;$ $\mathrm{HI} 90=0.10$ ) y SRMR $=0.05$. Además, los índices de modificación propuestos por el programa no mejoraban el ajuste del modelo.

\section{Fiabilidad}

La consistencia interna de la EPAAD medida a través del alfa de Cronbach fue de 0.93. La estabilidad temporal de la escala se evaluó a partir de una muestra de 54 deportistas que cumplimentaron la escala en dos ocasiones, tras un intervalo de ocho semanas. El alfa del pre-test fue de 0.93 y el alfa del post-test fue de 0.96. Además, la correlación test-retest fue de 0.73 , mostrando evidencias de estabilidad temporal.

\section{Validez predicitiva}

Para evaluar la validez concurrente se realizó un modelo de ecuaciones estructurales, donde se establecieron relaciones entre las siguientes variables: percepción de apoyo a la autonomía, percepción de autonomía, percepción de competencia, motivación intrínseca y emociones positivas.

Para analizar las diferentes relaciones entre las variables se utilizó el método de dos pasos sugerido por Anderson y Gerbing (1988). En primer lugar, se analizó el modelo de medida, ya que proporciona validez de constructo al instrumento. En segundo lugar, se analizó el modelo estructural con el objetivo de analizar las relaciones entre las distintas variables. La evaluación de ajuste del modelo se realizó con índices de ajuste absolutos y relativos. Tanto el modelo de medida como el modelo estructural fueron identificados, ya que todas las variables latentes tenían al menos dos indicadores, como sugieren McDonald y Ho (2002). Concretamente, las variables utilizadas fueron: el apoyo a la autonomía percibido, que se dividió en tres indicadores de cuatro ítems cada uno, las necesidades psicológicas básicas (percepción de autonomía y percepción de competencia), la motivación intrínseca (hacia el conocimiento, el logro y las experiencias estimulantes) y, por último, las emociones positivas, que se dividió en dos indicadores de dos ítems por factor.

\section{Step 1. Modelo de medida}

El modelo de medida establece relaciones bidireccionales entre todas la variables sometidas a estudio. Los índices de ajuste fueron los siguientes: $\chi^{2} / g l=1.52, \mathrm{IFI}=0.99, \mathrm{CFI}=0.99, \mathrm{RMSEA}=0.04$ $(\mathrm{LO} 90=0.02 ; \mathrm{HI} 90=0.06)$ y $\mathrm{SRMR}=0.03$.

\section{Step 2. Modelo de ecuaciones estructurales}

El modelo estructural pone a prueba un modelo hipotético posible de las relaciones entre las variables no observables, donde la percepción de apoyo a la autonomía predecía la percepción de autonomía y la percepción de competencia, y estas a su vez predecían la motivación intrínseca. Por último, la motivación intrínseca tenía un efecto positivo sobre las emociones positivas (véase Figura 1). Los índices de ajuste del modelo fueron los siguientes: $\chi^{2} / g l=2.36 ; \mathrm{CFI}=0.97$ IFI $=0.97$; RMSEA $=0.06($ LO $90=0.05 ;$ HI $90=0.08)$; SRMR $=0.07)$. Además, todos los parámetros fueron significativos $(p<0.01)$ y las covarianzas entre las variables fueron positivas. Tal y como se observa en la Figura 1, las relaciones entre percepción de apoyo a la autonomía y percepción de autonomía y de competencia fueron positivas ( $\beta=0.59$ y $\beta=0.55$, respectivamente) y significativas $(p<0.01)$. Las relaciones entre estas y la motivación intrínseca fueron positivas $(\beta=0.27$ у $\beta=0.38$, respectivamente) y significativas $(p<0.01)$ y, por último, la relación en la motivación intrínseca y las emociones positivas fue también positiva $(\beta=0.47)$ y significativa $(p<0.01)$. De acuerdo con estos resultados, se establece que el modelo se considera satisfactorio. Por último, es necesario destacar que el efecto indirecto de la percepción de apoyo a la autonomía en la motivación intrínseca y en las emociones positivas, es significativo con un nivel de confianza del $95 \%$. 


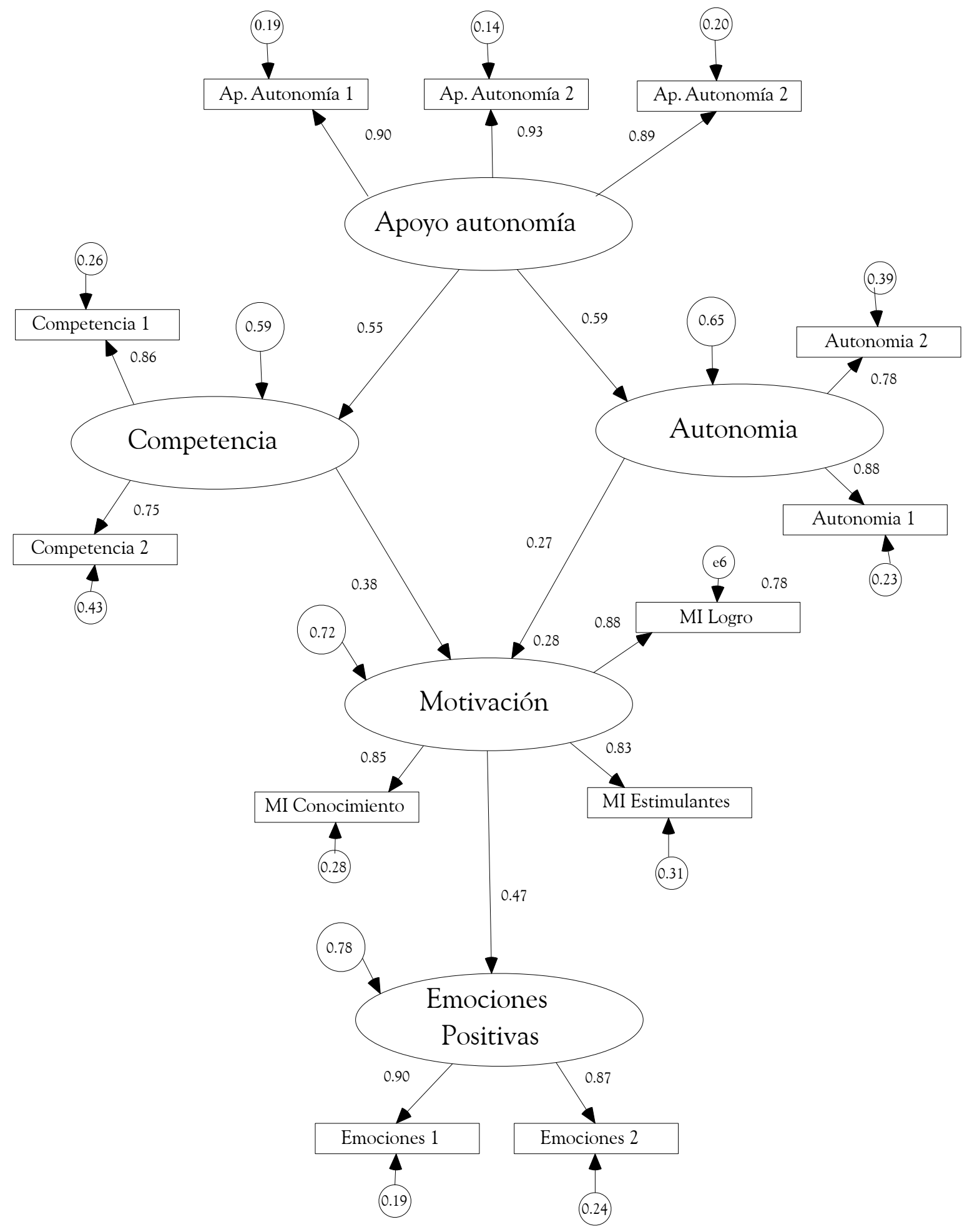

Figura 1. Modelo de ecuaciones estructurales.

Nota. Los errores estandarizados y la varianza residual se muestran dentro de los círculos pequeños. Fuente: elaboración propia. 


\section{Discusión}

El objetivo del presente estudio fue traducir al español y analizar las propiedades psicométricas de la escala EPAAD en el contexto deportivo.

Respecto a la validez de constructo, se analizó la estructura factorial de la EPAAD mediante un AFC. Los resultados confirmaron la estructura unifactorial propuesta inicialmente por Hagger et al. (2007) y fueron similares a los obtenidos por Gillet et al. (en prensa), en la validación francesa de la escala. Además, todos los pesos factoriales fueron estadísticamente significativos con valores estandarizados por encima de 0.50 , contribuyendo significativamente a la evaluación del constructo de interés, lo que corrobora la estructura unidimensional de la escala.

El análisis de la consistencia interna de la EPA$\mathrm{AD}$ mostró un valor superior al valor encontrado por Gillet et al. (en prensa) en su versión original, y similares a los encontrados por otros autores con escalas que evalúan la percepción del apoyo a la autonomía en contextos físico-deportivos (Álvarez et al., 2009; Moreno et al., 2008). Asi mismo, los resultados de la estabilidad temporal han sido aceptables, con valores superiores a los encontrados en el estudio realizado para la validación original de la escala (Gillet et al., en prensa).

Se tiene un modelo ajustado de acuerdo con el cual la percepción del apoyo a la autonomía predecía de manera positiva la percepción de autonomía y la percepción de competencia; estas, a su vez, predecían positivamente la motivación intrínseca y, por último, la motivación intrínseca tendría una influencia positiva sobre las emociones positivas, durante la práctica deportiva. Esta hipótesis es consistente con la secuencia propuesta por el modelo jerárquico de motivación intrínseca y extrínseca (Vallerand, 1997). En un contexto en el que el deportista siente que su entrenador le permite diferentes posibilidades a la hora de ejecutar una tarea, este se sentirá más autónomo, más libre en sus decisiones y más competente, durante la práctica deportiva, aumentando su motivación intrínseca, comprometiéndose en la actividad por el placer y satisfacción que se derivan de su práctica y por el aumento de sus conocimientos y éxitos relacionados con la práctica deportiva. Esto, a su vez, deriva en un incremento del bienestar hedónico del deportista, durante la actividad deportiva.

No obstante, se señalan algunas de las limitaciones en el presente estudio. En primer lugar, las evidencias de validez y fiabilidad deben ser consideradas como provisionales, dado que el tamaño de la muestra, sobre todo de mujeres, no ha sido amplio. Próximos estudios deberían comprobar efectos de género en una muestra mayor y analizar la invarianza por género. En segundo lugar, no se tuvo en cuenta el efecto que puede tener el hecho de que los deportistas contestaran a los instrumentos antes de empezar el entrenamiento, próximos estudios podrían tener en cuenta este efecto, evaluando tanto antes como después del entrenamiento. En tercer lugar, no se ha analizado la evidencia de validez concurrente respecto a otras escalas que midan la percepción de apoyo a la autonomía. En cuarto lugar, se debe recordar que este estudio es transversal, por lo que podría haber otros posibles modelos, y solo un estudio longitudinal permitiría establecer la dirección y las relaciones entre las variables (Hershberger, 2006).

Como conclusión, en general, los resultados mostraron que la EPAAD presenta evidencias preliminares de fiabilidad y de validez, en la misma línea de los obtenidos en la versión original (Gillet et al., en prensa), contando con unas propiedades psicométricas aceptables que permiten su utilización para evaluar la percepción del apoyo a la autonomía en el contexto deportivo, dentro del marco teórico de la teoría de la autodeterminación.

\section{Referencias}

Álvarez, M. S., Balaguer, I., Castillo, I. \& Duda, J. L. (2009). Coach autonomy support and quality of sport engagement in young soccer players. The Spanish Journal of Psychology, 12(1), 138-148.

Anderson, J. C. \& Gerbing, D. W. (1988). Structural equation modelling in practice: $\mathrm{A}$ review and recommended two-step approach. Psychological Bulletin, 103, 411-423. 
Baard, P. P., Deci, E. L. \& Ryan, R. M. (2004). Intrinsic need satisfaction: A motivational basic of performance and well-being in two work settings. Journal of Applied Social Psychology, 34, 2045-2068.

Balaguer, I., Castillo, I., Duda, J. L. \& Tomás, I. (2009). Análisis de las propiedades psicométricas de la versión española del Cuestionario de Clima en el Deporte. Revista de Psicología del Deporte, 18, 73-83.

Bandura, A. (1997). Self-efficacy: The exercise of control. New York: Freeman.

Blais, M. R. \& Vallerand, R. J. (1991). Échelle de Perception d'Autodétermination dans les Domaines de Vie (ÉPADV-16). Manuscrito no publicado. Universitè du Québec.

Blanchard, C. M., Amiot, C. E., Perreault, S., Vallerand, R. J. \& Provencher, P. (2009). Cohesiveness and psychological needs: Their effects on selfdetermination and athletes' subjective well-being. Psychology of Sport E Exercise, 10, 545-551.

Byrne, B. M. (2001). Structural equation modeling with AMOS: Basic concepts, applications, and programming. Mahwah, NJ: Lawrence Erlbaum.

Curran, P. J., West, S. G. \& Finch, J. F. (1996). The robustness of test statistics to nonnormality and specification error in confirmatory factor analysis. Psychological Methods, 1(1), 16-29.

Deci, E. L., Connell, J. P. \& Ryan, R. M. (1989). Selfdetermination in a work organization. Journal of Applied Psychology, 74, 580-590.

Deci, E. L. \& Ryan, R. M. (1985). Intrinsic motivation and self-determination in human behavior. New York: Plenum.

Deci, E. L. \& Ryan, R. M. (1991). A motivational approach to self: Integration in personality. En R. A. Dienstbier (Ed.), Nebraska Symposium on Motivation: Perspectives on motivation (Vol. 38, pp. 237288). Lincoln, NE: University of Nebraska Press.

Deci, E. L., Ryan, R. M., Gagné, M., Leone, D. R., Usunov, J. \& Kornazheva, B. P. (2001). Need satisfaction, motivation, and well-being in the work organizations of a former eastern bloc country: A cross cultural study of self-determination. Personality and Social Psychology Bulletin, 27, 930-942.

Gagné, M. \& Deci, E. L. (2005). Self-determination theory and work motivation. Journal of Organizational Behavior, 26, 331-362.
Gillet, N., Rosnet, E. \& Vallerand, R. J. (2008). Développement d'une échelle de satisfaction des besoins fondamentaux en contexte sportif. Canadian Journal of Behavioural Science, 40(4), 230-237.

Gillet, N., Vallerand, R. J., Paty, E., Gobancé, L. \& Berjot, S. (en prensa). French validation and adaptation of the Perceived Autonomy Support Scale for Exercise Settings to the sport context. International Journal of Sport and Exercise Psychology, 8(2).

Hackman, J. R. \& Oldham, G. R. (1975). Development of the Job Diagnostic Survey. Journal of Applied Psychology, 60, 159-170.

Hagger, M. S., Chatzisarantis, N. L. D., Hein, V., Pihu, M., Soós, I. \& Karsai, I. (2007). The Perceived Autonomy Support Scale for Exercise Settings (PASSES): Development, validity and cross-cultural invariance in young people. Psychology of Sport and Exercise, 8, 632-653.

Hambleton, R. K. (1996). Adaptación de test para su uso en diferentes idiomas y culturas: fuentes de error, posibles soluciones y directrices prácticas. En J. Muñiz (Ed.), Psicometría (pp. 67-89). Madrid: Universitas.

Hershberger, S. L. (2006). The problem of equivalent structural models. En G. R. Hancock \& R. O. Mueller (Eds.), Structural equation modeling: A second course (pp. 13-41). Greenwich: IAP.

Lynn, M. (1986). Determination and quantification of content validity. Nursing Research, 35, 382-85.

Mageau, G. A. \& Vallerand, R. J. (2003). The coachathlete relationship: A motivational model. Journal of Sports Sciences, 21, 883-904.

McDonald, R. P. \& Ho, R. M. (2002). Principles and practice in reporting structural equation analyses. Psychological Methods, 7, 64-82.

Moreno, J. A., Parra, N. \& González-Cutre, D. (2008). Influencia del apoyo a la autonomía, las metas sociales y la relación con los demás sobre la desmotivación en educación física. Psicothema, 20(4), 636-641.

Ntoumanis, N. (2005). A prospective study of participation in optional school physical education using a self-determination theory framework. Journal of Educational Psychology, 97, 444-453.

Núñez, J. L., Martín-Albo, J., Navarro J. G. \& González, V. M. (2006). Preliminary validation of a Spanish 
version of the sport motivation scale. Perceptual and Motor Skills, 102, 919-930.

Pelletier, L. G., Fortier, M. S., Vallerand, R. J. \& Brière, N. M. (2001). Associations among perceived autonomy support, forms of self-regulation, and persistence: A prospective study. Motivation and Emotion, 25, 279-306.

Pelletier, L. G., Fortier, M. S., Vallerand, R. J., Tuson, K. M., Brière, N. M. \& Blais, M. R. (1995). Toward a new measure of intrinsic motivation, extrinsic motivation, and amotivation in sports: The Sport Motivation Scale (SMS). Journal of Sport and Exercise Psychology, 17, 35-54.

Petrides, K. V. \& Furnham, A. (2003). Trait emotional intelligence: Behavioural validation in two studies of emotion recognition and reactivity to mood induction. European Journal of Personality, 17, 39-75.

Philippe, F. L. \& Vallerand, R. J. (2008). Environments do affect motivation and psychological adjustment: A test of self-determination theory in a natural setting. Motivation and Emotion, 32, 81-89.

Reeve, J. (2002). Self-determination theory applied to educational settings. En E. L. Deci \& R. M. Ryan (Eds.), Handbook of self-determination research (pp. 183-203). Rochester, NY: University of Rochester Press.

Reeve, J. \& Deci, E. L. (1996). Elements of the competitive situation that affect intrinsic motivation. Personality and Social Psychology Bulletin, 22, 24-33.

Ryan, R. M. \& Deci, E. L. (2000). Self-determination theory and the facilitation of intrinsic motivation, social development, and well-being. American Psychologist, 55, 68-78.
Vallerand, R. J. (1997). Toward a hierarchical model of intrinsic and extrinsic motivation. En M. Zanna (Ed.), Advances in experimental social psychology (pp. 271-360). New York: Academic Press.

Vallerand, R. J., Blais, M. R., Brière, N. M. \& Pelletier, L. G. (1989). Construction et validation de l'Échelle de Motivation en Éducation (EME). Canadian Journal of Behavioral Sciences, 21, 323-349.

Vallerand, R. J. \& Losier, G. (1999). An integrative analysis of intrinsic and extrinsic motivation in sport. Journal of Applied Sport Psychology, 11, 142-169.

Waterman, A. S., Schwartz, S. J. \& Conti, R. (2008). The implications of two conceptions of happiness (hedonic enjoyment and eudaimonia) for the understanding of intrinsic motivation. Journal of Happiness Studies, 9, 41-79.

Williams, G. C. \& Deci, E. L. (1996). Internalization of biopsychosocial values by medical students: A test of self-determination theory. Journal of Personality and Social Psychology, 70, 767-779.

Williams, G. C., Freedman, Z. R. \& Deci, E. L. (1998). Supporting autonomy to motivate glucose control in patients with diabetes. Diabetes Care, 21, 1644-1651.

Williams, G. C., Grow, V. M., Freedman, Z., Ryan, R. M. \& Deci, E. L. (1996). Motivational predictors of weight loss and weight-loss maintenance. Journal of Personality and Social Psychology, 70, 115-126.

Wong, E. H. \& Wiest, D. J. (1999). Adolescent depression: Link to academic coping and perceive autonomy support. Education, 119, 668-673. 